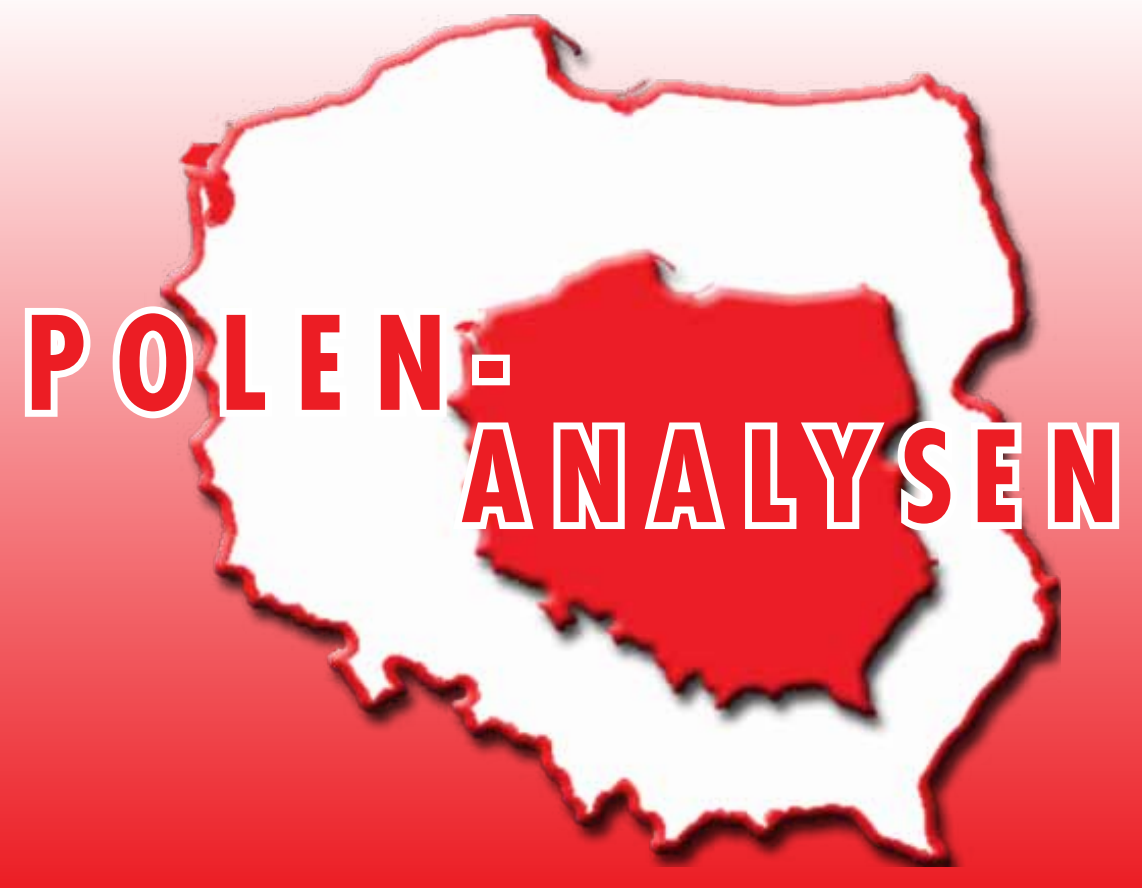

www.laender-analysen.de/polen

\title{
DAS RENTENSYSTEM
}

aNALYSE

Das Rentensystem in Polen - zehn Jahre nach der Reform auf dem Prüfstand

Urszula Banaszczak-Soroka, Breslau

- TABELLEN UND GRAFIKEN

Daten zum Rentensystem

- UMFRAGE

Meinungen zum Rentensystem

CHRONIK

Vom 4. bis zum 17. Mai 2010 


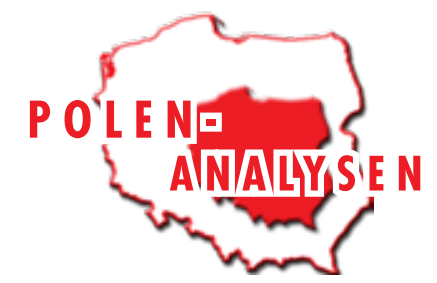

Analyse

\section{Das Rentensystem in Polen - zehn Jahre nach der Reform auf dem Prüfstand}

Urszula Banaszczak-Soroka, Breslau

\section{Zusammenfassung}

Das polnische Rentenversicherungssystem wurde 1999 reformiert. Wesentlicher Bestandteil der Reform war die Streuung der Möglichkeiten (»drei Säulen«), für die Rente einzuzahlen und infolgedessen die Diversifizierung der Finanzierungsquellen für zukünftige Rentenauszahlungen. Die Organisation des Rentensystems sollte allen Bürgern maximale soziale Sicherheit garantieren. Die völlig neuen Erfahrungen im Zusammenhang mit der gegenwärtigen Finanzkrise ließen die bisher unbemerkten Nachteile des Rentensystems zutage treten. Die Nutznießer der Reform sind nicht die zukünftigen Rentner, sondern die privaten Allgemeinen Rentenversicherungen. Aber auch die diskutierten Novellierungsvorschläge lösen nicht das Problem der in Polen relativ kurzen Lebensarbeitszeit und der dramatischen Veränderung der Alterspyramide. In den nächsten 20 Jahren wird sich die Bevölkerungszahl aufgrund der sinkenden Geburtenrate und aufgrund von Migrationsprozessen systematisch verringern und gleichzeitig nimmt die Lebenserwartung weiter zu. Auf diese Herausforderung muss das Rentensystem noch Antworten finden.

1 999 wurde in Polen das Rentenversicherungssystem reformiert. Die Reform betraf alle Geburtsjahrgänge ab 1949, wobei für die Jahrgänge bis 1968 die Möglichkeit bestand, in dem alten System zu bleiben. Die Reform des Rentensystems war angesichts der alternden Gesellschaft und der gleichzeitig zunehmenden Lebenserwartung notwendig geworden, was zu der Einschätzung geführt hatte, dass das alte Rentensystem nunmehr unzulänglich geworden sei. Es funktionierte nach dem Grundsatz, dass alle berufstätigen Personen für den Unterhalt der aus dem Arbeitsleben ausgeschiedenen Generationen verantwortlich sind, und basierte auf der Auszahlung der Rentenzahlungen durch die staatliche Sozialversicherungsanstalt (Zakład Ubezpieczeń Społecznych - ZUS). Im Staatshaushalt fehlen jedoch die notwendigen Mittel, die Rentenzahlungen zu tätigen. Wesentlicher Bestandteil der Reform war daher die Diversifizierung der Möglichkeiten, für die Rente einzuzahlen und infolgedessen die Diversifizierung der Finanzierungsquellen für zukünftige Rentenauszahlungen. Dies sollte in der Weise umgesetzt werden, dass von den Versicherten selbst die Verantwortung für ihre wirtschaftliche Situation im Alter übernommen wird. Das neue Rentensystem besteht aus drei "Säulen«: Mit der »ersten Säule« wird der Versicherte gegen Armut im Rentenalter abgesichert, die "zweite Säule« stellt einen ähnlichen Lebensstandard wie während der Berufstätigkeit sicher und die »dritte Säule» stellt einen höheren Lebensstandard im Rentenalter in Aussicht (Zusatzrentenversicherung).

Die »erste Säule« ist obligatorisch, sie umfasst alle Arbeitnehmer und wird von der ZUS organisiert, die den Sozialversicherungsfonds (Fundusz Ubezpieczeń Społecznych - FUS) verwaltet. Der FUS ist ein öffentlicher, zweckgebundener Fonds, der Geldmittel, darunter Rentenbeiträge, aus eindeutig bestimmten Quellen für eindeutig bestimmte Zwecke anhäuft. Die Höhe des Rentenbeitrags legt der Staat im Rahmen der Sozialpolitik fest. Zurzeit liegt er bei 19,52 \% des Bruttoeinkommens. Arbeitnehmer und Arbeitgeber übernehmen jeweils die Hälfte des Betrags. 12,22\% des monatlich abgeführten Gesamtbetrags werden auf dem individuellen Konto des Versicherten bei der ZUS gutgeschrieben, 7,3\% werden an die "zweite Säule« des Rentensystems überwiesen, die ebenfalls obligatorisch ist und alle Berufstätigen umfasst und die von den Offenen Rentenfonds (Otwarte Fundusze Emerytalne - OFE) organisiert wird (Ende 2009 gab es in Polen 14 OFE).

Das individuelle Konto des Versicherten bei der ZUS dient nur der Registrierung der eingehenden Beiträge. Da der FUS als zweckgebundener Fonds nicht in vorhandene Finanzinstrumente investieren kann, vermehrt er das eingehende Geld auch nicht. Die Finanzmittel, die sich aus den Beitragszahlungen ergeben, werden sofort in Form verschiedener Leistungen weiterverteilt, so dass es sich bei dem vom Versicherten angehäuften Kapital um ein fiktives Kapital handelt. Es ist nur "auf dem Papier" vorhanden und wird jährlich um den realen Anstieg von Preisen und Leistungen valorisiert.

Die monatliche Rentenauszahlung aus der "ersten Säule« der ZUS ergibt sich aus dem Rentenkapital dividiert durch einen demografischen Faktor. Das Rentenkapital ist abhängig vom Zeitpunkt des Renteneintritts. Mit der Länge der Lebensarbeitszeit erhöht 


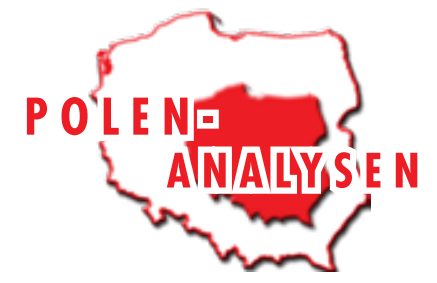

sich das Rentenkapital, da es sich um die Summe der valorisierten Beiträge handelt, die seit 1999 auf dem individuellen Konto des Versicherten registriert sind. Der demografische Faktor, der am 31. März jeden Jahres vom Statistischen Hauptamt (Główny Urząd Statystyczny - GUS) bekanntgegeben wird, ist die in Monaten ausgedrückte durchschnittliche Lebensdauer nach dem Renteneintritt; sie wird für Frauen und Männer einheitlich angesetzt. Diesen Teil der Rentenzahlung garantiert der Fiskus.

Für die Organisation der "zweiten Säule« zeichnen die OFE verantwortlich, die von privaten Allgemeinen Rentenversicherungen (Powszechne Towarzystwa Emerytalne-PTE) gebildet und verwaltet werden (jede PTE verwaltet einen OFE). Grundlegendes Ziel der OFE ist die Anhäufung von Geldmitteln (präziser die Übernahme dieser Mittel von der ZUS) und deren Anlage mit dem Ziel, sie den Mitgliedern des Fonds nach Erreichen des Rentenalters auszuzahlen (genauer: sie der ZUS für die Auszahlung zu übertragen). Die Zugehörigkeit zu den OFE ist für die arbeitende Bevölkerung ebenfalls obligatorisch. Jeder entscheidet sich für einen Fonds; für die Entscheidung hat der Betroffene sieben Tage nach Unterzeichnung des ersten Arbeitsvertrags Zeit. Ist keine Entscheidung getroffen worden, wird von der ZUS per Losverfahren ein OFE bestimmt. Ende 2009 umfassten die OFE knapp 14,3 Mio. Personen.

Die OFE sind ein Bestandteil des Kapitalrentensystems. Der Kapitalcharakter beruht auf der Investition der angehäuften Beiträge in die Finanzmärkte. Daher ist die Höhe dieses Teils der Rente, der aus dem jeweiligen OFE gezahlt wird, nicht nur von der Höhe der Beiträge (also des Einkommens) und der Dauer der Beitragsleistungen (der Arbeitszeit) abhängig, sondern auch und vielleicht vor allem von den Anlageergebnissen des jeweiligen OFE und der makroökonomischen Situation. Eine Ergänzung der offiziellen Formen der Rentenversicherung sind Arbeitnehmer Rentenprogramme (Pracownicze Programy Emerytalne - PPE) sowie Individuelle Rentenkonten (Indywidualne Konta Emerytalne IKE). Eine solche Ergänzung der offiziellen Formen wird unter den Begriff der »dritten Säule« des Rentensystems gefasst. Sie ist freiwillig und im Fall der PPE eine gruppenspezifische, im Fall der IKE eine individuelle Anhäufung von Mitteln für die zukünftige Rente. Beide Formen der Ergänzung sind wenig verbreitet.

\section{Die ersten zehn Jahre des neuen Rentensystems}

Das zehnjährige Bestehen der Rentenreform fiel in die Zeit der Finanzkrise und enthüllte viele Nachteile des reformierten Rentensystems. Es hat sich herausgestellt, dass die Nutznießer der Reform nicht die zukünftigen Rentner sind, sondern die PTE. Als die PTE ihren Inhabern Gewinne einbrachten sowie eine überdurchschnittliche Rentabilität (siehe Grafik auf S. 6) und damit einhergehend Dividenden (fast 2,5 Mrd. Zloty in zehn Jahren), begannen die OFE die Aktiva ihrer Fondsmitglieder zu verlieren. Vom 31. Dezember 2007 bis 31. Dezember 2008 verloren die Rentenfonds mindestens $21 \mathrm{Mrd}$. Zloty. Auf diese Weise gingen fast alle Gelder, die ihnen aus den Rentenbeiträgen im Jahr 2008 von der ZUS weitergeleitet worden waren, unwiderruflich verloren.

Diese Situation hatte unmittelbaren Einfluss auf die von den Fondsmitgliedern erzielte Rentabilität.

Wie die statistischen Daten zeigen, hat sich das Rentenkapital umgerechnet in Verrechnungseinheiten infolge von Fehlern der verantwortlichen Verwalter und/ oder negativer wirtschaftlicher Entwicklungen deutlich verringert, was sich unmittelbar auf das Niveau der künftigen Rente auswirkt. Nach dem 1. Quartal 2009 begannen die OFE langsam, die erlittenen Verluste aufzuholen (s. Grafik auf S. 7 oben).

Die Organisation des Rentensystems sollte allen Bürgern maximale soziale Sicherheit garantieren. Es wurde von der Annahme ausgegangen, dass man mindestens den Betrag, den man in den Fonds spart, auch im Rentenalter erhält. Es muss nun allerdings deutlich hervorgehoben werden, dass die OFE im Namen der Versicherten investieren (investieren als langfristige, mit Risiko belastete Allokation wirtschaftlicher Ressourcen) und nicht sparen (sparen als Sicherstellung der finanziellen Mittel ohne Risiko) und dabei nicht nur Gewinne, sondern auch Verluste aufgrund einer falschen Investitionspolitik oder einer negativen wirtschaftlichen Situation erzielen. So trägt der Berufstätige allein das volle Risiko, das von den angehäuften Aktiva, der wirtschaftlichen Situation im Land und der Art und Weise der Bewirtschaftung der Aktiva ausgeht.

Die völlig neuen Erfahrungen im Zusammenhang mit der gegenwärtigen Finanzkrise ließen die bisher unbemerkten Nachteile des Rentensystems zutage treten und lösten eine Welle breiter Diskussionen aus, an denen sich die Regierung und andere Politiker, Vertreter der Wissenschaft und der Gesellschaft und andere Experten beteiligen. Dabei kamen auch extreme, manchmal exotische Ansichten zu Wort. Ein Beispiel für letzteres ist der Diskussionsbeitrag des stellvertretenden Ministerpräsidenten und Wirtschaftsministers Waldemar Pawlak von Ende April 2010, der eine Senkung der Rentenbeiträge auf 120 Zloty monatlich vorschlägt und jedem Rentner in der Zukunft eine Rentenauszahlung in Höhe 


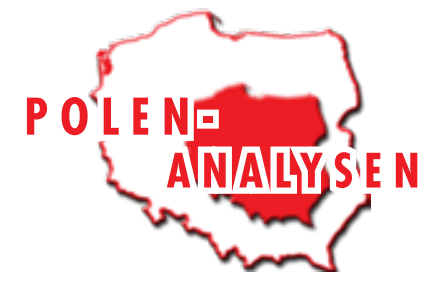

von mindestens 1.200 Zloty von der ZUS. (Nach Meinung von Experten müsste ein Rentner dann mindestens 70 Jahren arbeiten, sollte die defizitäre ZUS und damit der Staatshaushalt nicht belastet werden.) Eine andere Ansicht stellte Andrzej Sadowski, der Gründer und Vizepräsident des unabhängigen Wirtschaftsinstituts Adam Smith-Zentrum, vor, der eine Reform in Anlehnung an das kanadische Modell vorschlägt. Die selbstverwaltete Wirtschaftskammer für Rentenversicherungen (Izba Gospodarcza Towarzystw Emerytalnych) dagegen ist für die Beibehaltung des gegenwärtigen Systems. Es überwiegt jedoch die gemäßigte Einschätzung, dass das bestehende Rentensystem einiger Korrekturen unterzogen werden sollte. Im Folgenden sollen ausgewählte Aspekte dargestellt werden.

\section{Ausgewählte Korrekturvorschläge zum Rentensystem}

Die Beobachtung der OFE während der Krise und die Tatsache, dass zum ersten Mal Renten aus den OFE ausgezahlt werden, zwang die Regierung und das Parlament, erste schnelle Entscheidungen über Änderungen des geltenden Gesetzes über die OFE zu treffen. Eine wesentliche Änderung betraf die Höhe der von den OFE einbehaltenen Verwaltungsgebühr. Diese Gebühr verringerte jeden monatlichen Beitrag des Fondsmitglieds, der von der ZUS überwiesen wurde, und minderte so dessen Aktiva auf seinem individuellen Konto. 1999 wurde diese Gebühr gesetzlich im Bereich von $7 \%$ festgelegt; sie sollte bis 2014 auf das Niveau von 3,5\% sinken. Die Verwaltungsgebühr, die der Konkurrenz zwischen den Fonds dienen sollte, wurde nie in dieser Weise eingesetzt. In der Praxis haben alle OFE, bis auf zwei Ausnahmen, die höchstmögliche Gebühr erhoben. In Hinblick auf das Wohl des Versicherten nahm die Regierung 2009 eine Novellierung des Gesetzes für die OFE vor und legte die Höhe der Verwaltungsgebühr auf maximal 3,5\% fest. Diese Gebühr ist die größte und nicht die einzige Belastung für das Fondsmitglied. Von den Brutto-Aktiva der Fondsmitglieder werden noch weitere Verpflichtungen der OFE bestritten, die u. a. gegenüber den PTE (für die Verwaltung der Aktiva), Finanzberatungsunternehmen (Provisionen und Gebühren für die Empfehlungen von An- und Verkauf von an der Wertpapierbörse notierten Finanzinstrumenten) oder dem Depositar (für die Aufbewahrung von Wertpapieren) eingegangen werden.

Eine grundlegende Veränderung betraf die Art und Weise der Auszahlung der Renten. Im Rahmen der Reform des Rentensystems sollte noch eine Instanz entstehen, die Rentenanstalten (Zakłady Emerytalne -
ZE), die ebenfalls privat bewirtschaftet werden sollten, so wie die OFE. Sie sollten von den OFE die Aktiva derjenigen übermittelt bekommen, die in Rente gehen, sie sollten mit diesen Geldern wirtschaften und die Renten auszahlen bzw. anderen Verpflichtungen nachkommen, die die Rentner gegenüber den betreffenden Begünstigten eingegangen sind. Jeder sollte das Recht haben, eine lebenslange Rente auszuwählen, was im Grunde bedeutet zu kaufen (d. h. zum zweiten Mal für die Verwaltung der Aktiva und die Organisation der Auszahlungen zu zahlen), ohne die Möglichkeit zu haben, die ZE zu wechseln. Dieses Problem ist sowohl gesellschaftlich als auch politisch so umstritten, dass es bis heute nicht endgültig gelöst ist. Den Rentnern - eigentlich den einigen tausend Rentnerinnen, die die Berechtigungen im neuen Rentensystem erworben haben - zahlt zurzeit die ZUS die Leistungen, sowohl aus eigenen Mitteln als auch aus den von den OFE überwiesenen, denn mit dem Gesetz über die Kapitalrenten vom 21. November 2008 wurde ein eigentümliches Provisorium eingeführt, das der ZUS neue Befugnisse erteilt. Die Befürworter sind der Ansicht, dass es auf diese Weise billiger für den Rentner wird, die Gegner meinen, dass dies eine Negierung der Reformidee sei, mit dem Ziel, die Gelder der Rentner in die staatliche und in gewisser Weise politische ZUS zurückzuführen.

Die genannten Änderungen lösen nicht die vielen Kontroversen, die das derzeitige Rentensystem hervorruft. Sich mit der Frage der Lebensarbeitszeit zu beschäftigen, ist das Problem, das zu akzeptieren der Gesellschaft am schwersten fällt. In den nächsten 20 Jahren wird sich die Bevölkerungszahl im Land aufgrund der sinkenden Geburtenrate und aufgrund von Migrationsprozessen systematisch verringern. Gleichzeitig verlängert sich die Lebenserwartung. Gegenwärtig leben Frauen durchschnittlich 80 Jahre und Männer 71,3 Jahre (im Vergleich zu den 1950er Jahren ist dies eine Verlängerung der Lebenserwartung bei Frauen um 18 Jahre und bei Männern um 15 Jahre). Nach den Prognosen wird die Lebenserwartung noch um ca. drei bzw. sechs Jahre steigen. Dies bedeutet eine bedeutende Veränderung hinsichtlich der Altersstruktur der Bevölkerung, d. h. der Anstieg des Anteils der Generationen im arbeitsfähigen Alter (von 6,2 Mio. im Jahr 2008 auf 9,6 Mio. im Jahr 2035) bei gleichzeitigem Rückgang des prozentualen Anteils von Kindern und Jugendlichen, was sich in der Konsequenz - plus die Migration - im Rückgang der Anzahl der Menschen im arbeitsfähigen Alter widerspiegeln wird (von 24,6 Mio. im Jahr 2008 auf 20,7 Mio. im Jahr 2035). Derart wesentliche Veränderungen in der Bevölkerungsstruktur werden sich 


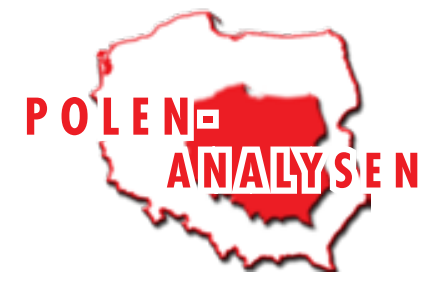

stark auf den Arbeitsmarkt auswirken, darüber hinaus auf die Beitragseinzahlungen in die ZUS und die Steuereinnahmen des Staates, so dass die Frage des Unterhalts der älteren Menschen, die länger leben (für sich genommen eine positive Entwicklung), immer dringlicher wird. Nach den geltenden Vorschriften erreichen Frauen das Rentenalter mit 60 und Männer mit 65 Jahren. Das Durchschnittsalter derjenigen, die im Jahr 2005 Rente erhalten haben, betrug bei den Frauen 56 Jahre (im Jahr 2008 56,2 Jahre) und bei den Männern 58,4 Jahre (im Jahr 2008 61,1 Jahre). Hier zeigt sich zwar eine steigende Tendenz, sie ist aber noch weit von der gesetzlich formulierten Grenze entfernt.

Nach Meinung vieler Wirtschaftsexperten sollte das Rentenalter nicht nur zum Wohl des zukünftigen Rentners, sondern auch für die Sicherheit des Staates angehoben werden; zumindest sollte das Renteneintrittsalter von Männern und Frauen auf 65 Jahre angeglichen und Personen, die bereits im Rentenstand sind, wieder für den Arbeitsmarkt aktiviert werden. Im Februar 2010 hat das Zentrum zur Erforschung der öffentlichen Meinung (Centrum Badania Opinii Społecznej - CBOS) eine Umfrage zur Akzeptanz der Anhebung des Renteneintrittsalters durchgeführt. $74 \%$ der Polen waren dagegen.

Für die Aktiva der Rentenfonds wurden gesetzlich Anlagegrenzen (quantitative Beschränkungen) hinsichtlich dessen eingeführt, dass sich der Fonds an einem maximalen Grad an Sicherheit und Rentabilität der betreffenden Anlagen unter Berücksichtigung des Risikograds, den die einzelnen Instrumente bergen, orientieren soll (s. Tabelle auf S. 7 unten). Die vom Staat auferlegte Investitionspolitik und das eigentümliche Paradox, das die Fondsanlagen erfüllen sollen (maximale Sicherheit und gleichzeitig maximale Rentabilität der Anlagen) verursacht, dass sich die OFE auf dem Markt konservativ verhalten. Dies belegt die Zusammensetzung des Investitionsportfolios der Fonds mit einem eindeutigen Übergewicht an Staatsanleihen. Eine solche Investitionspolitik garantiert keine zufrieden stellenden Umsätze in einer "normalen", nicht von Krisen erschütterten Finanzsituation (s. Grafik auf S. 8 oben).

Die Ursachen dieser Investitionsregeln lassen sich auch am allzu flachen, regulierten Kapitalmarkt beobachten. Das Angebot anderer Finanzinstrumente als der Staatsanleihen ist verglichen mit der von den OFE erzeugten Nachfrage beschränkt. Die Kapitalisierung der an der Wertpapierbörse notierten einheimischen Gesellschaften (regulierter Markt) betrug in der Zeit des größten Rückgangs ca. 230 Mrd. Zloty und Ende 2009

421,2 Mrd. Zloty. Die vom Ministerium für Arbeit und Sozialpolitik vorbereitete Novelle des Gesetzes über die OFE beinhaltet die Einführung von Änderungen bei den Grenzen und Richtungen der Anlage der Aktiva der OFE abhängig vom Fondstyp und schlägt zwei Unterfonds vor: Unterfonds A soll Personen bis zum 50. Lebensjahr vorbehalten sein, er verhält sich aggressiver und hat eine breitere Auswahl an Finanzinstrumenten; Unterfonds B ist für Personen ab dem 50. Lebensjahr bestimmt, er ist sicher und legt vor allem in Anlagen des Staates oder der Selbstverwaltungen an. Auf die Investitionspolitik hat auch die makroökonomische Situation Einfluss. Die Verschlechterung der wirtschaftlichen Situation, Krisen an den Finanzmärkten, jede Baisse auf dem Aktienmarkt und der beschränkte Zufluss neuer Anlageinstrumente an die Börse wirkt sich auf die Höhe der künftigen Leistung aus, umso mehr als Phasen von Baissen und Haussen, Krisen und dynamischen Wirtschaftswachstums periodisch auftreten und unterschiedlich lang dauern. Bei Eintritt in die Rente während einer Hausse ist die Rente höher als während einer Baisse.

Diese dargelegten Aspekte der Diskussion um Änderungen im Rentensystem sind nicht das letzte Wort in der seit einigen Jahren scharf geführten Auseinandersetzung. Es tauchen immer wieder neue Vorschläge aus dem Ministerium für Arbeit und Sozialpolitik auf, die sofort von verschiedenen gesellschaftlichen oder mit den OFE verbundenen Gruppierungen kritisiert werden, zum Beispiel:

- die Senkung der Beiträge, die den OFE von der ZUS überwiesen werden, von 7,3\% auf $3 \%$;

- die Möglichkeit, Verträge mit den OFE zu kündigen und die dort angehäuften Mittel komplett dem FUS zu übertragen; evtl. die Übergabe nur eines Teils der Mittel an den FUS, die im Rahmen der "zweiten Säule« angehäuft worden sind, ohne die Notwendigkeit, den Vertrag mit der OFE zu kündigen (dies betrifft Frauen im Alter von 55 Jahren und Männer im Alter von 60 Jahren);

- die Möglichkeit einer einmaligen Auszahlung der auf dem Konto der OFE angehäuften Mittel, und zwar dann, wenn die von den Mitgliedern der OFE erreichte Rente aus der "ersten Säule« die niedrigste Rente um mehr als das Doppelte übersteigt. Wenn jedoch die Rente aus der »ersten Säule« nicht mehr als das Doppelte der niedrigsten Rente beträgt, dann vermehren die im Rahmen der "zweiten Säule« angehäuften Mittel als einmaliger Beitrag das Konto des Versicherten beim FUS, was automatisch die Erhöhung der Leistung aus der »ersten Säule« verursacht. 


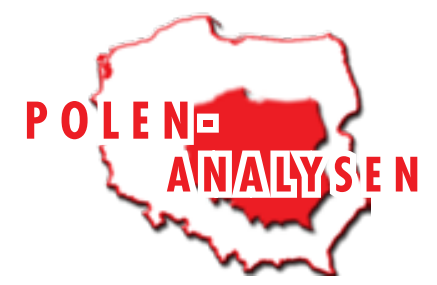

Die Reform des Rentensystems ist zweifellos politisch und gesellschaftlich sehr schwierig. Die unternommenen Versuche einer erneuten Reform bzw. der Korrektur wecken aus gutem Grund große Emotionen. Im weiteren Verlauf ist eine Umorganisation des Rentensystems für die Landwirte in der Kasse der Landwirtschaftlichen Sozialversicherung (Kasa Rolniczego Ubezpieczenia Społecznego - KRUS) wünschenswert sowie des Rentensystems für die uniformierten Staatsdiener und die Bergleute, darüber hinaus eine Umorganisation des vorzeitigen Ruhestands usw. Dies wird teilweise sogar radikale Änderungen erfordern. Auf das Rentensystem darf nicht nur duch das Prisma der OFE geblickt werden. Die Änderungen sollten umfassend sein und das gesamte, zurzeit ausufernde und instabile Rentensystem neu ordnen.

Übersetzung aus dem Polnischen: Silke Plate

Über die Autorin

Dr. Urszula Banaszczak-Soroka ist Wirtschaftswissenschaftlerin am Institut für Wirtschaftswissenschaften der Universität Wroclaw in Breslau. Vorher hatte sie leitende Funktionen im Bankwesen inne und war Aufsichtsratsmitglied von Börsengesellschaften sowie Richterin am schiedsrichterlichen Börsengericht der Wertpapierbörse in Warschau. Schwerpunkt ihres Interesses ist der Kapitalmarkt. Neben zahlreichen Veröffentlichungen ist ihr 2008 erschienenes Buch »Institutionen und Teilnehmer des Kapitalmarkts« [»Instytucje i uczestnicy rynku kapitałowego«, Warszawa 2008] zu nennen.

\section{Tabellen und Grafiken}

\section{Daten zum Rentensystem}

\section{Gewinn und Rentabilität der Allgemeinen Rentenversicherungen (PTE)}

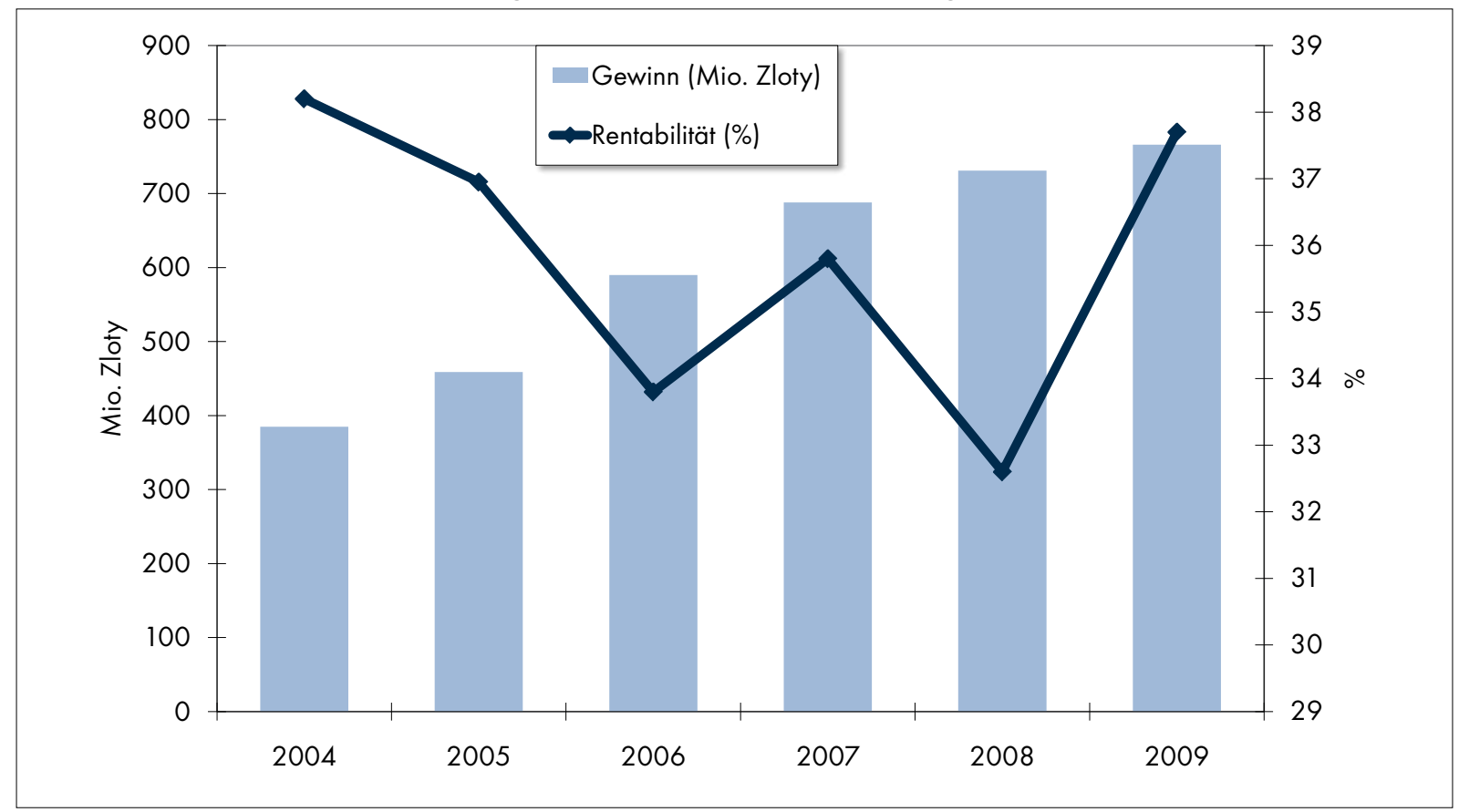

Quelle: Zusammenstellung der Autorin Urszula Banaszczak-Soroka 


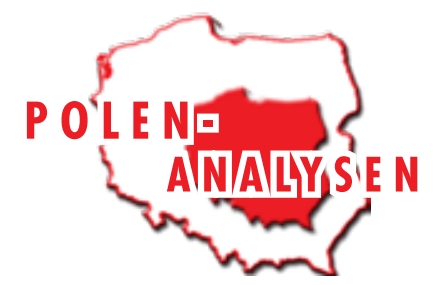

Jährliches und dreijähriges Wachstum der Offenen Rentenfonds (OFE) in \% (gewogener Mittelwert)

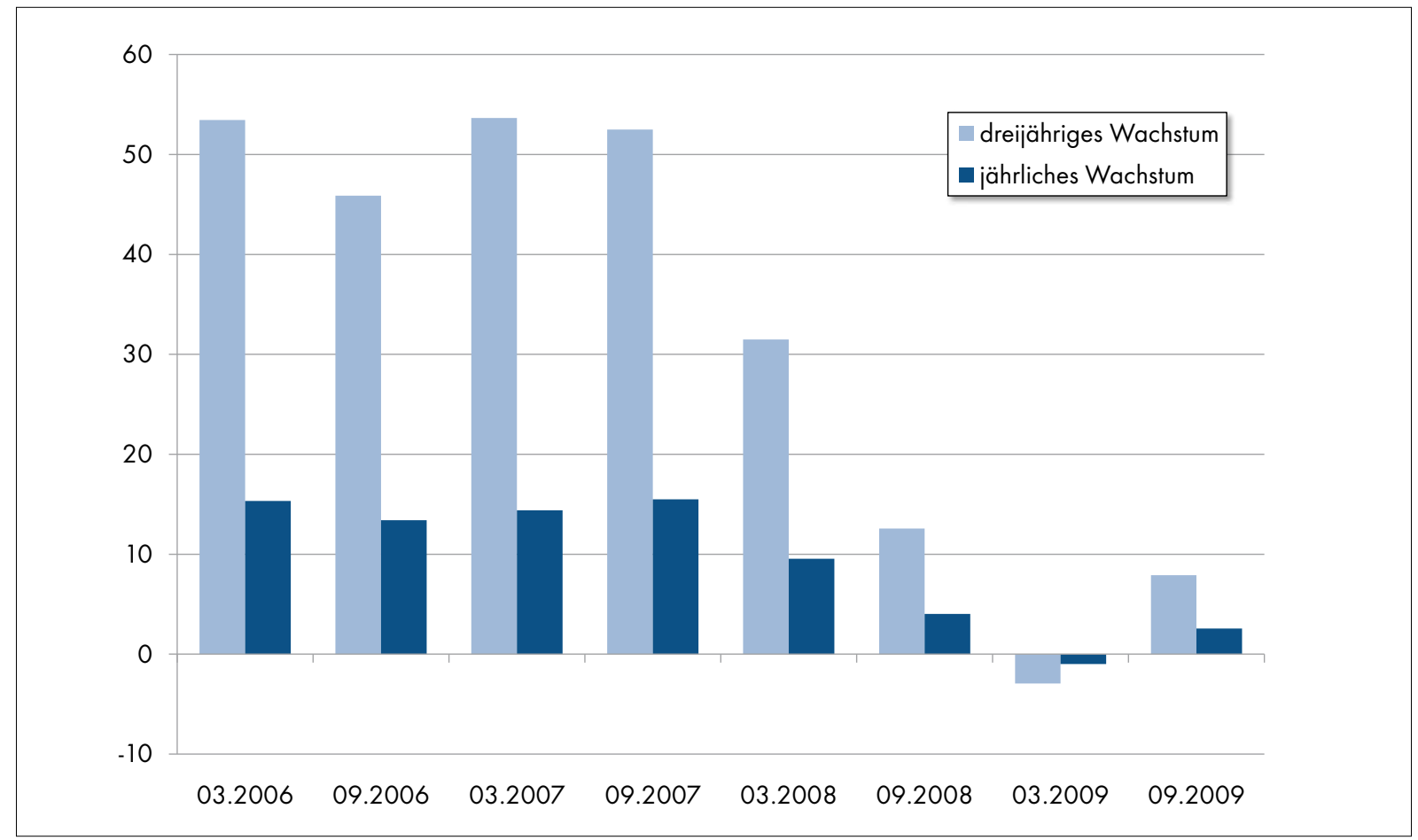

Quelle: Zusammenstellung der Autorin Urszula Banaszczak-Soroka

\section{Anlagerichtungen und -beschränkungen der Offenen Rentenfonds (OFE) - Beispiele}

\begin{tabular}{|l|c|}
\hline $\begin{array}{l}\text { Wertpapier eines einzelnen Emittenten; Wertpapiere, die nicht } \\
\text { am öffentlichen Markt notiert sind; ausländische Wertpapiere }\end{array}$ & $5 \%$ \\
$\begin{array}{l}\text { Wertpapiere, die von den PTE* emittiert werden; Immobilien; } \\
\text { derivative Finanzinstrumente }\end{array}$ & verboten \\
börsennotierte Aktien & $40 \%$ \\
$\begin{array}{l}\text { außerbörslich notierte Aktien und Aktien der Nationalen } \\
\text { Investitionsfonds }\end{array}$ & $10 \%$ \\
Anteilseinheiten an offenen Investitionsfonds & $15 \%$ \\
Bankdeposita & $20 \%$ \\
kommunale Anleihen, für den öffentlichen Umlauf zugelassen & $15 \%$ \\
$\begin{array}{l}\text { Staatsanleihen, Schatzanweisungen, von der Polnischen } \\
\text { Nationalbank emittierte Wertpapiere }\end{array}$ & ohne Beschränkung \\
*Allgemeine Rentenversicherungen & \\
Quelle: Zusammenstellung der Autorin Urszula Banaszczak-Soroka & \\
\hline
\end{tabular}




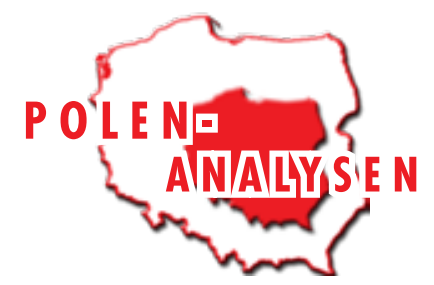

Investitionsportfolio der Offenen Rentenfonds (OFE) 2001-2009 (Quartalsdaten in Mio. Zloty)

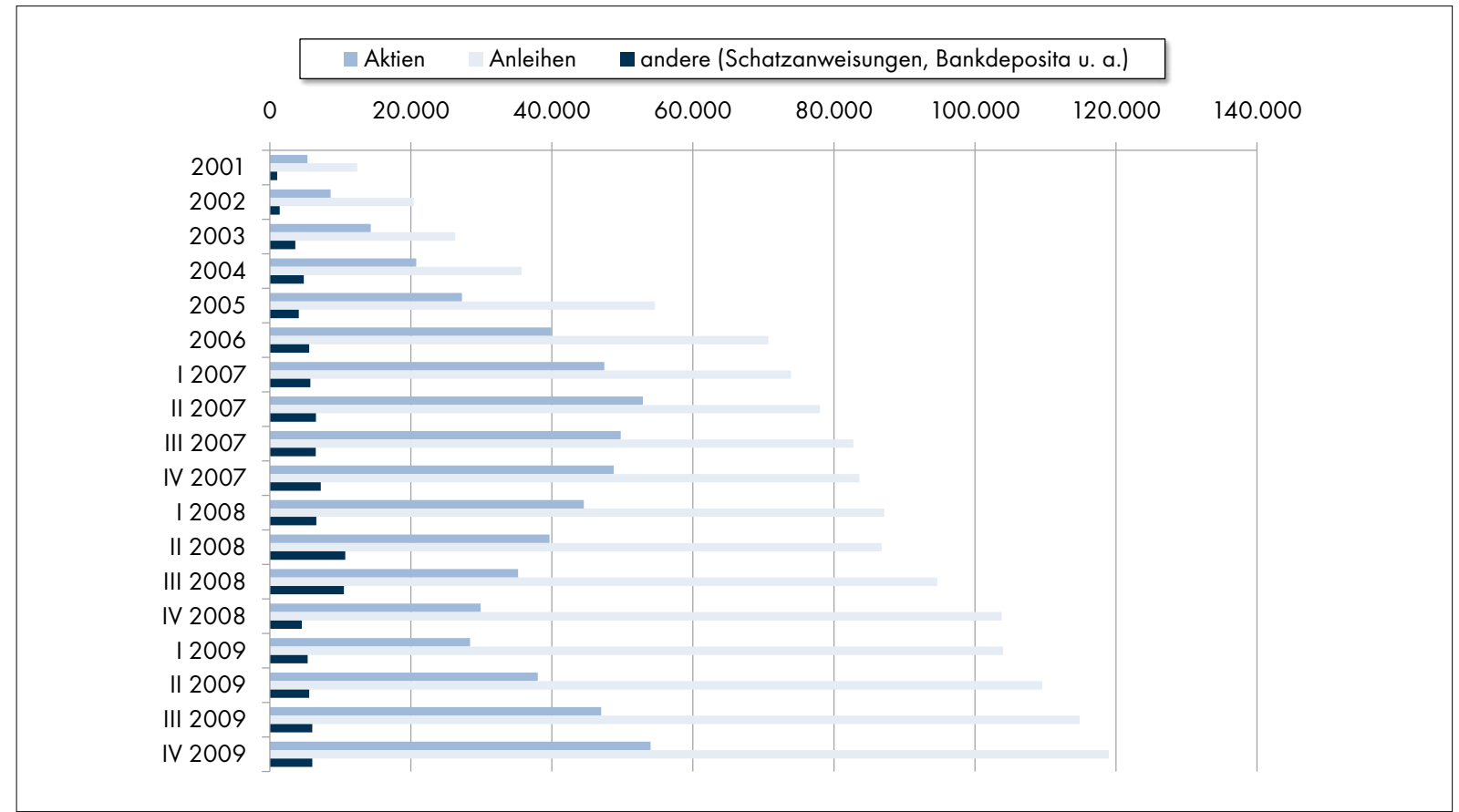

Quelle: Zusammenstellung der Autorin Urszula Banaszczak-Soroka

\section{Umfrage}

\section{Meinungen zum Rentensystem}

Sind Sie zufrieden/unzufrieden mit dem Funktionieren unseres Rentensystems? (\%)

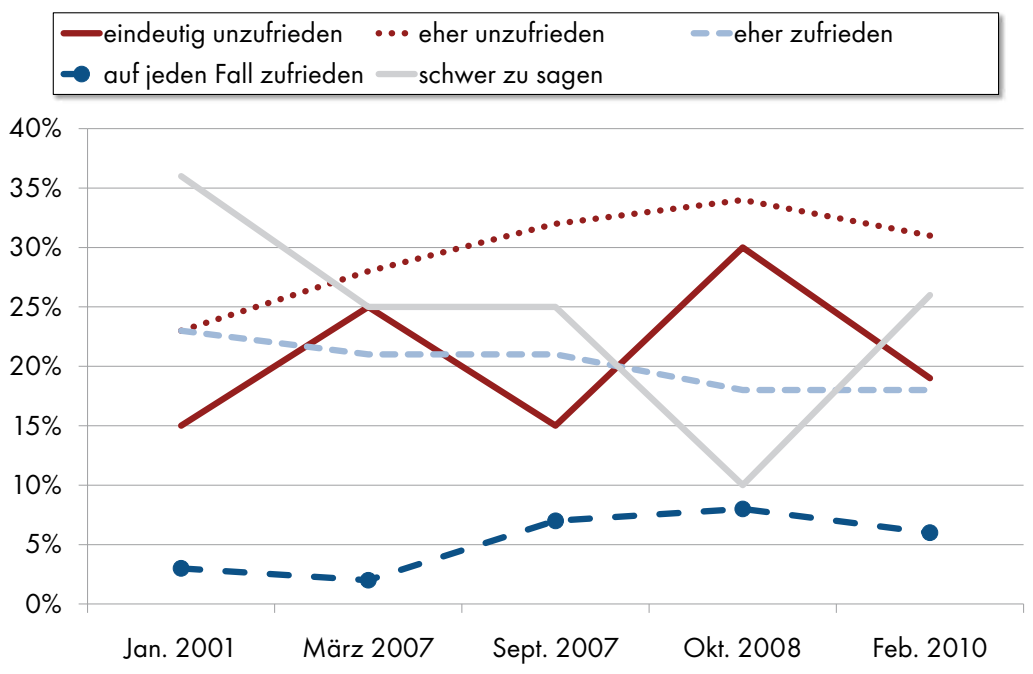

CBOS, BS/41/2010: Oceny proponowanych zmian w systemie emerytalnym [Bewertungen der vorgeschlagenen Änderungen des Rentensystems]. Warszawa 03/2010. www.cbos.pl 


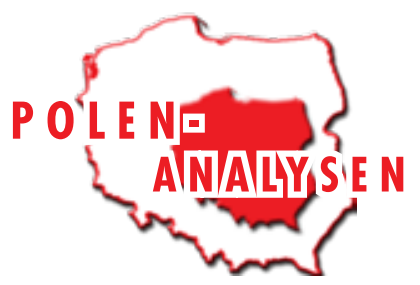

Welche Lösung halten Sie für die beste?

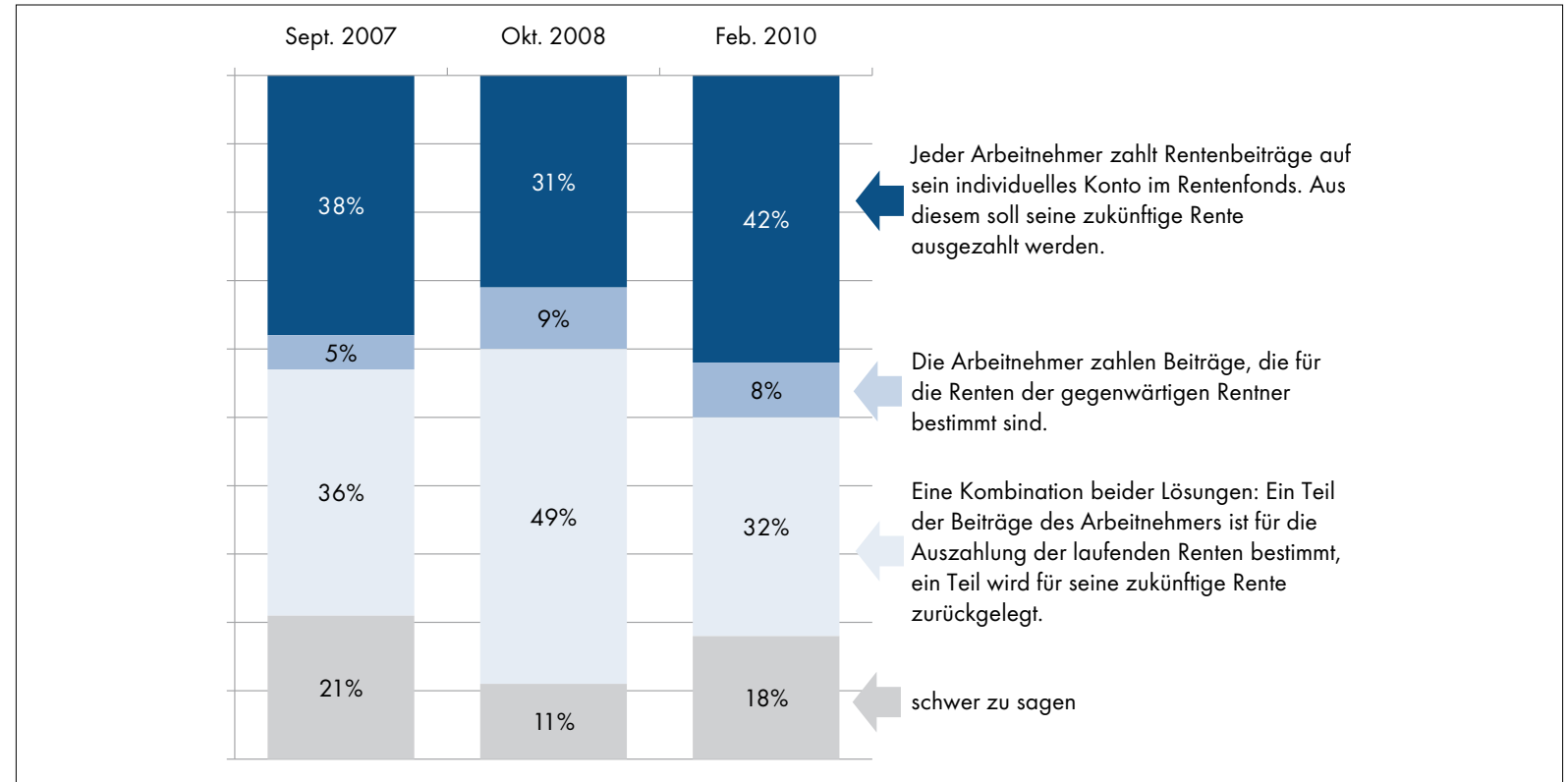

CBOS, BS/41/2010: Oceny proponowanych zmian w systemie emerytalnym [Bewertungen der vorgeschlagenen Änderungen des Rentensystems]. Warszawa 03/2010. www.cbos.pl

Im Jahr 1999 wurde in Polen eine Rentenreform durchgeführt. Das alte System, in dem aus den Beiträgen der Arbeitnehmer die laufenden Rentenzahlungen getätigt wurden, wurde durch ein neues System ersetzt, in dem jeder Arbeitnehmer sein individuelles Rentenkonto hat, auf das er seine Rentenbeiträge einzahlt. In dem neuen System ist die Höhe der Rente von der Höhe der auf dem individuellen Konto gutgeschriebenen Beiträge abhängig.

Für wen ist Ihrer Meinung nach diese Änderung gewinnbringend?

\begin{tabular}{|c|c|c|c|c|c|c|c|}
\hline & \multicolumn{2}{|c|}{ Öffentliche Finanzen } & \multicolumn{2}{|c|}{ Arbeitnehmer } & \multicolumn{2}{|c|}{$\begin{array}{l}\text { Renter, die nach dem } \\
\text { neuen Rentensystem } \\
\text { ihre Leistungen erhalten } \\
\text { werden }\end{array}$} & \multirow{2}{*}{$\begin{array}{c}\text { Rentner, die bereits } \\
\text { jetzt nach dem neuen } \\
\text { System ihre Leis- } \\
\text { tungen erhalten }\end{array}$} \\
\hline & 2008 & 2010 & 2008 & 2010 & 2008 & 2010 & \\
\hline $\begin{array}{l}\text { eindeutig } \\
\text { gewinn- } \\
\text { bringend }\end{array}$ & $13 \%$ & $6 \%$ & $6 \%$ & $5 \%$ & $4 \%$ & $3 \%$ & $1 \%$ \\
\hline $\begin{array}{l}\text { eher gewinn- } \\
\text { bringend }\end{array}$ & $32 \%$ & $28 \%$ & $28 \%$ & $31 \%$ & $20 \%$ & $25 \%$ & $16 \%$ \\
\hline $\begin{array}{l}\text { eher nicht } \\
\text { gewinn- } \\
\text { bringend }\end{array}$ & $13 \%$ & $15 \%$ & $27 \%$ & $21 \%$ & $25 \%$ & $22 \%$ & $25 \%$ \\
\hline $\begin{array}{l}\text { auf keinen } \\
\text { Fall gewinn- } \\
\text { bringend }\end{array}$ & $4 \%$ & $5 \%$ & $11 \%$ & $8 \%$ & $17 \%$ & $12 \%$ & $12 \%$ \\
\hline $\begin{array}{l}\text { ohne Bedeu- } \\
\text { tung }\end{array}$ & $5 \%$ & $7 \%$ & $3 \%$ & $3 \%$ & $3 \%$ & $3 \%$ & $8 \%$ \\
\hline $\begin{array}{l}\text { schwer zu } \\
\text { sagen }\end{array}$ & $33 \%$ & $39 \%$ & $25 \%$ & $32 \%$ & $31 \%$ & $35 \%$ & $39 \%$ \\
\hline
\end{tabular}

CBOS, BS/41/2010: Oceny proponowanych zmian w systemie emerytalnym [Bewertungen der vorgeschlagenen Änderungen des Rentensystems]. Warszawa 03/2010. www.cbos.pl 


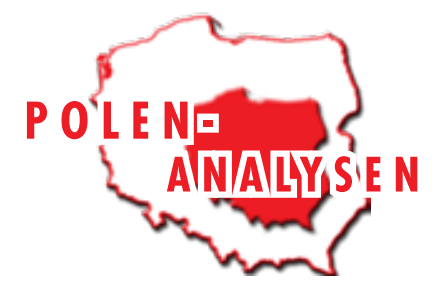

\section{Wie hoch wird Ihrer Meinung nach in der Zukunft, beispielsweise in 15 Jahren, die Rente bezogen auf das Einkommen sein?}

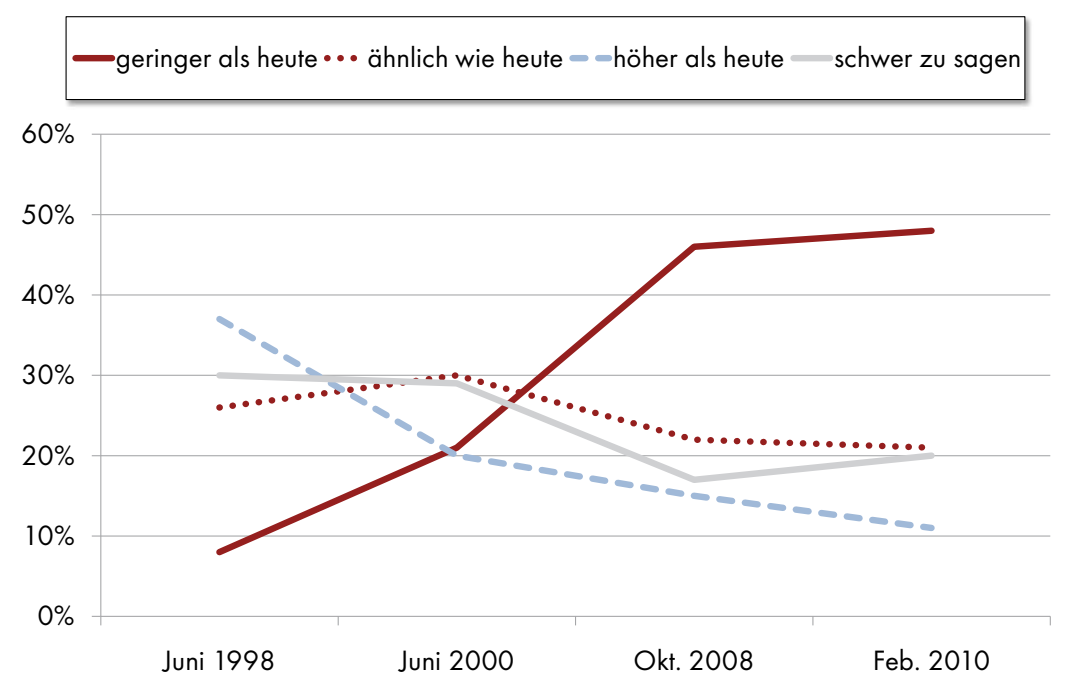

CBOS, BS/41/2010: Oceny proponowanych zmian w systemie emerytalnym [Bewertungen der vorgeschlagenen Änderungen des Rentensystems]. Warszawa 03/2010. www.cbos.pl

\section{Chronik}

\section{Vom 4. bis zum 17. Mai 2010}

\begin{tabular}{|l|l|}
\hline 04.05 .2010 & $\begin{array}{l}\text { Geistliche, Intellektuelle und Künstler rufen dazu auf, am 9. Mai, dem 65. Jahrestag des Endes des Zweiten } \\
\text { Weltkriegs, die Gräber der Angehörigen der Roten Armee, die sich zu Tausenden in Polen befinden, mit Kerzen } \\
\text { und Blumen zu schmücken. Dies soll ein Zeichen für eine neue Öffnung zwischen Polen und Russland und } \\
\text { eine Reaktion auf die Solidaritätsbekundungen der russischen Öffentlichkeit gegenüber Polen anlässlich der } \\
\text { Flugzeugkatastrophe von Smolensk sein, bei der hochrangige Vertreter der Politik und des öffentlichen Lebens } \\
\text { Polens im April tödlich verunglückt waren. }\end{array}$ \\
\hline 05.05 .2010 & $\begin{array}{l}\text { Der Wahlkampfleiter des Präsidentschaftskandidaten der Bürgerplattform (Platforma Obywatelska - PO), } \\
\text { Sławomir Nowak, teilt mit, dass der ehemalige Ministerpräsident Tadeusz Mazowiecki, der ehemalige Staats- } \\
\text { präsident Lech Wałęsa und der ehemalige Außenminister und gegenwärtige Staatssekretär für den Interna- } \\
\text { tionalen Dialog beim Ministerpräsidenten, Władysław Bartoszewski, dem Ehrenkomitee zur Unterstützung } \\
\text { des PO-Kandidaten, Bronisław Komorowski, angehören. }\end{array}$ \\
\hline 06.05 .2010 & $\begin{array}{l}\text { Die Mehrheit der Sejmabgeordneten stimmt gegen den Antrag der Fraktion von Recht und Gerechtigkeit (Prawo } \\
\text { i Sprawiedliwość- PiS), über eine Resolution von PiS abzustimmen, in der gefordert wird, dass sich die polnische } \\
\text { Regierung an die russische Regierung wenden solle, um die Aufklärung der Ursachen der Flugzeugkatastrophe } \\
\text { von Smolensk übertragen zu bekommen. }\end{array}$ \\
\hline 06.05.2010 & $\begin{array}{l}\text { Ministerpräsident Donald Tusk hebt die gute Zusammenarbeit zwischen Russland und Polen bei der Aufklärung } \\
\text { der Ursachen des Flugzeugunglücks von Smolensk hervor. Russland habe schnell auf die Bitte der polnischen } \\
\text { Seite reagiert, den Unglücksort angemessen zu sichern, und komme den Bitten nach Information und Zugang } \\
\text { bereitwillig nach. Tusk reagiert damit auf die Kritik von Politikern und Medienvertretern, dass die Aufklärung } \\
\text { chaotisch und inkompetent verlaufe. }\end{array}$ \\
\hline
\end{tabular}




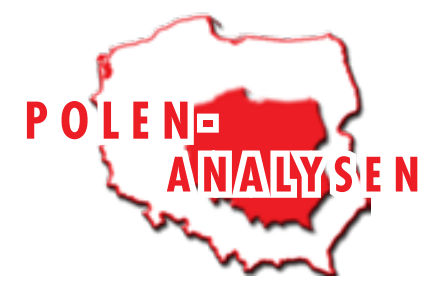

\begin{tabular}{|c|c|}
\hline 07.05 .2010 & $\begin{array}{l}\text { Der Fraktionsvorsitzende der Bürgerplattform (Platforma Obywatelska - PO), Grzegorz Schetyna, teilt mit, } \\
\text { dass die derzeitige Geschäftsführerin der Stiftung für deutsch-polnische Zusammenarbeit, Irena Lipowicz, als } \\
\text { Kandidatin der PO für das Amt des Bürgerrechtsbeauftragten nominiert wurde. Der/die Bürgerrechtsbeauf- } \\
\text { tragte wird vom Sejm gewählt. }\end{array}$ \\
\hline 08.05 .2010 & $\begin{array}{l}\text { Bei einem Treffen im Rahmen der Feierlichkeiten zum 65. Jahrestag des Endes des Zweiten Weltkriegs in Moskau } \\
\text { übergibt der russische Präsident Dmitri Medwedew dem amtierenden Staatspräsidenten Bronisław Komorowski } \\
67 \text { Bände bereits veröffentlichter Ermittlungsakten aus den Jahren } 1990 \text { bis } 2004 \text { zum Massaker von Katyn, } \\
\text { bei dem } 1940 \text { Tausende polnische Offiziere und Zivilpersonen vom NKWD ermordet worden waren. Darüber } \\
\text { hinaus kündigt er an, dass weitere Dokumente von der Geheimhaltung befreit werden sollen. }\end{array}$ \\
\hline 11.05 .2010 & $\begin{array}{l}\text { Die Delegation des Europäischen Parlaments für die Beziehungen zu Russland bezeichnet in einer Erklärung } \\
\text { zum Gedenken der Opfer des Flugzeugabsturzes von Smolensk das Massaker von Katyn im April } 1940 \text { als } \\
\text { „Kriegsverbrechen vom Charakter eines Völkermords«. Europaabgeordnete von Recht und Gerechtigkeit (Prawo } \\
\text { i Sprawiedliwość - PiS) kündigen an, sich um eine Debatte über das Massaker von Katyn im Europäischen } \\
\text { Parlament zu bemühen. }\end{array}$ \\
\hline 11.05 .2010 & $\begin{array}{l}\text { Der Pressesprecher von Recht und Gerechtigkeit (Prawo i Sprawiedliwość - PiS), Mariusz Błaszczak, teilt mit, } \\
\text { dass die ehemalige Solidarność-Aktivistin Zofia Romaszewska PiS-Kandidatin für das Amt des Bürgerrechts- } \\
\text { beauftragten sei. }\end{array}$ \\
\hline 12.05 .2010 & $\begin{array}{l}\text { In einem Radiointerview spricht sich Finanzminister Jacek Rostowski für den Beitritt Polens zur Eurozone } \\
\text { frühestens im Jahr } 2015 \text { aus. }\end{array}$ \\
\hline 13.05 .2010 & $\begin{array}{l}\text { Ministerpräsident Donald Tusk wird in Aachen der Internationale Karlspreis zu Aachen verliehen. Die Laudatio } \\
\text { hält Bundeskanzlerin Angela Merkel. Der Karlspreis ist eine Auszeichnung für besondere Verdienste für die } \\
\text { europäische Einigung. }\end{array}$ \\
\hline 13.05 .2010 & $\begin{array}{l}\text { In der Generalversammlung der Vereinten Nationen in New York wird Polen für die Amtszeit von 2010-2013 } \\
\text { in den UN-Menschenrechtsrat gewählt. Der Menschenrechtsrat mit Sitz in Genf hat } 2006 \text { die Menschenrechts- } \\
\text { kommission der Vereinten Nationen abgelöst und zählt } 47 \text { Mitglieder. }\end{array}$ \\
\hline 15.05 .2010 & $\begin{array}{l}\text { Der ehemalige Staatspräsident Aleksander Kwaśniewski wird Vorstandsvorsitzender der ukrainischen Stiftung } \\
\text { Yalta European Strategy, der er vorher drei Jahre als Mitglied des Vorstands angehört hat. Die Stiftung wurde } \\
2006 \text { von dem ukrainischen Oligarchen Victor Pinchuk gegründet und hat zum Ziel, die europäische Integration } \\
\text { der Ukraine zu befördern. }\end{array}$ \\
\hline 16.05 .2010 & $\begin{array}{l}\text { Der Kandidat der Demokratischen Linksallianz (Sojusz Lewicy Demokratycznej-SLD) für das Amt des Staats- } \\
\text { präsidenten, Grzegorz Napieralski, spricht sich für eine gemeinsame öffentliche Debatte der Kandidaten von } \\
\text { SLD, Bürgerplattform (Platforma Obywatelska - PO), Recht und Gerechtigkeit (Prawo i Sprawiedliwość - PiS) } \\
\text { und Polnischer Bauernpartei (Polskie Stronnictwo Ludowe - PSL) aus. }\end{array}$ \\
\hline 17.05 .2010 & $\begin{array}{l}\text { Vor der Einberufung des Rates für Nationale Sicherheit (Rada Bezpieczeństwa Narodowego) hebt der amtierende } \\
\text { Staatspräsident Bronisław Komorowski hervor, dass er das Gremium als Forum der politischen Zusammenarbeit } \\
\text { nach den Präsidentschaftswahlen versteht und ihm zum ersten Mal Vertreter der Opposition angehören sollen. } \\
\text { Der Rat für Nationale Sicherheit ist beim Staatspräsidenten angesiedelt und hat beratende Funktion in Fragen } \\
\text { der inneren und äußeren Sicherheit. }\end{array}$ \\
\hline
\end{tabular}




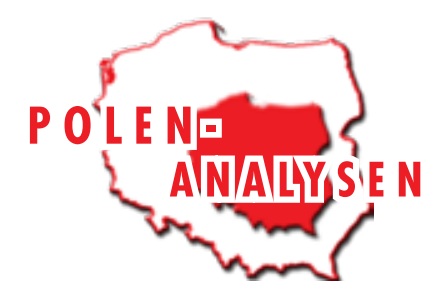

Über die Polen-Analysen

Die Polen-Analysen erscheinen zweimal monatlich als E-Mail-Dienst. Sie werden gemeinsam vom Deutschen PolenInstitut Darmstadt, der Bremer Forschungsstelle Osteuropa und der Deutschen Gesellschaft für Osteuropakunde herausgegeben.

Ein Archiv der Polen-Analysen finden Sie im Internet unter www.laender-analysen.de/polen

Kostenloses Abonnement unter http://www.deutsches-polen-institut.de/Newsletter/subscribe.php

\section{Deutsches Polen-Institut Darmstadt}

Das Deutsche Polen-Institut Darmstadt (DPI) ist ein Forschungs-, Informations-, und Veranstaltungszentrum für polnische Kultur, Geschichte, Politik, Gesellschaft und die deutsch-polnischen Beziehungen, die sich im Kontext der europäischen Integration entwickeln. Das seit März 1980 aktive und bis 1997 von Gründungsdirektor Karl Dedecius geleitete Institut ist eine Gemeinschaftsgründung der Stadt Darmstadt, der Länder Hessen und Rheinland-Pfalz sowie des Bundes. Seit 1987 ist die Trägerschaft auf die Kultusminister der Länder ausgedehnt. Einen wesentlichen Beitrag zur Verwirklichung der Institutsziele leisten private Stiftungen. Das DPI hat satzungsgemäß die Aufgabe, durch seine Arbeit zur Vertiefung der gegenseitigen Kenntnisse des kulturellen, geistigen und gesellschaftlichen Lebens von Polen und Deutschen beizutragen.

Ziel der Vermittlertätigkeit des DPI ist es, „die zu interessieren, auf die es politisch, wirtschaftlich, gesellschaftlich und kulturell im deutsch-polnischen Verhältnis ankommt" (Leitlinien 1997). Es geht um die Entscheider und Multiplikatoren in Politik, Kultur, Bildung, Verwaltung, Medien und Wirtschaft und, wesentlich stärker ausgeprägt als bisher, um das Hineinwirken in Wissenschaft, Forschung und Bildung.

Derzeit bemüht sich das DPI in Kooperation mit den verstreuten Orten wissenschaftlicher Polen-Kompetenz an deutschen Hochschulen und Forschungsinstituten verstärkt darum, ausgehend von einer Bestandsaufnahme deutscher Polen-Forschung Ort wissenschaftlicher Forschung und verbindendes, vernetzendes und kooperierendes Zentrum zu werden. Ausgangspunkt der Neuausrichtung ist die kaum mehr kontrollierbare Dynamik des Rückbaus der Ressourcen der wissenschaftlichen Polen-Kompetenz in den unterschiedlichen Disziplinen. Mit der über 55.000 Bände zählenden multidisziplinären Fachbibliothek für Polen mit einer einzigartigen Sammlung polnischer Literatur in der Originalsprache und in deutscher Übersetzung ist das DPI bereits ein geschätzter Ort der Recherche und des wissenschaftlichen Arbeitens. (www.deutsches-polen-institut.de)

\section{Forschungsstelle Osteuropa an der Universität Bremen}

1982 gegründet, widmet sich die Forschungsstelle Osteuropa an der Universität Bremen kulturellen und gesellschaftlichen Entwicklungen der Länder Ost- und Ostmitteleuropas in Zeitgeschichte und Gegenwart. Die Forschungsstelle besitzt in ihrem Archiv eine einzigartige Sammlung alternativer Kulturgüter und unabhängiger Texte aus den ehemaligen sozialistischen Ländern. Darunter befindet sich auch eine umfangreiche Sammlung des "Zweiten Umlaufs", die das Schrifttum und Dokumente unabhängiger Initiativen und gesellschaftlicher Gruppen in Polen aus der Zeit von 1976 bis zum Umbruch umfasst. Neben ausführlicher individueller Forschung zu Dissens und Gesellschaft im Sozialismus, leitet die Forschungsstelle seit Januar 2007 ein gemeinsames Projekt mit einem Verbund von internationalen Forschungsinstituten zum Thema "Das andere Osteuropa - die 1960er bis 1980er Jahre, Dissens in Politik und Gesellschaft, Alternativen in der Kultur. Beiträge zu einer vergleichenden Zeitgeschichte«, welches von der VolkswagenStiftung finanziert wird.

Im Bereich der post-sozialistischen Gesellschaften sind in den letzten Jahren umfangreiche Forschungsprojekte durchgeführt worden, deren Schwerpunkte auf politischen Entscheidungsprozessen, Wirtschaftskultur und der EU-Osterweiterung lagen. Eine der Hauptaufgaben der Forschungsstelle ist die Information der interessierten Öffentlichkeit. Dazu gehören unter anderem regelmäßige E-Mail-Informationsdienste mit fast 15.000 Abonnenten in Politik, Wirtschaft und den Medien.

Mit ihrer in Deutschland einzigartigen Sammlung von Publikationen zu Osteuropa ist die Forschungsstelle eine Anlaufstelle sowohl für Wissenschaftler als auch für die interessierte Öffentlichkeit. In der Bibliothek sind derzeit neben anderen breit angelegten Beständen allein aus Polen ca. 300 laufende Periodika zugänglich. Die Bestände werden in Datenbanken systematisch erfasst. (www.forschungsstelle.uni-bremen.de)

Die Meinungen, die in den Polen-Analysen geäußert werden, geben ausschließlich die Auffassung der Autoren wieder. Abdruck und sonstige publizistische Nutzung sind nach Rücksprache mit der Redaktion gestattet.

Redaktion: Prof. Dr. Dieter Bingen (Darmstadt), Silke Plate, M.A. (Bremen) Technische Gestaltung: Matthias Neumann

Polen-Analysen-Layout: Cengiz Kibaroglu, Matthias Neumann

Die Polen-Analysen werden im Rahmen der Datenbank World Affairs Online (WAO) ausgewertet und sind im Portal IREON www.ireon-portal.de recherchierbar.

ISSN 1863-9712 @ 2010 by Deutsches Polen-Institut Darmstadt und Forschungsstelle Osteuropa, Bremen

Kontakt: Dr. Andrzej Kaluza, Presse- und Öffentlichkeitsarbeit, Deutsches Polen-Institut, Mathildenhöhweg 2,

D-64287 Darmstadt, Tel.: 06151/4985-13, Fax: 06151/4985-10, E-Mail: polen-analysen@dpi-da.de, Internet: www.laender-analysen.de/polen 